
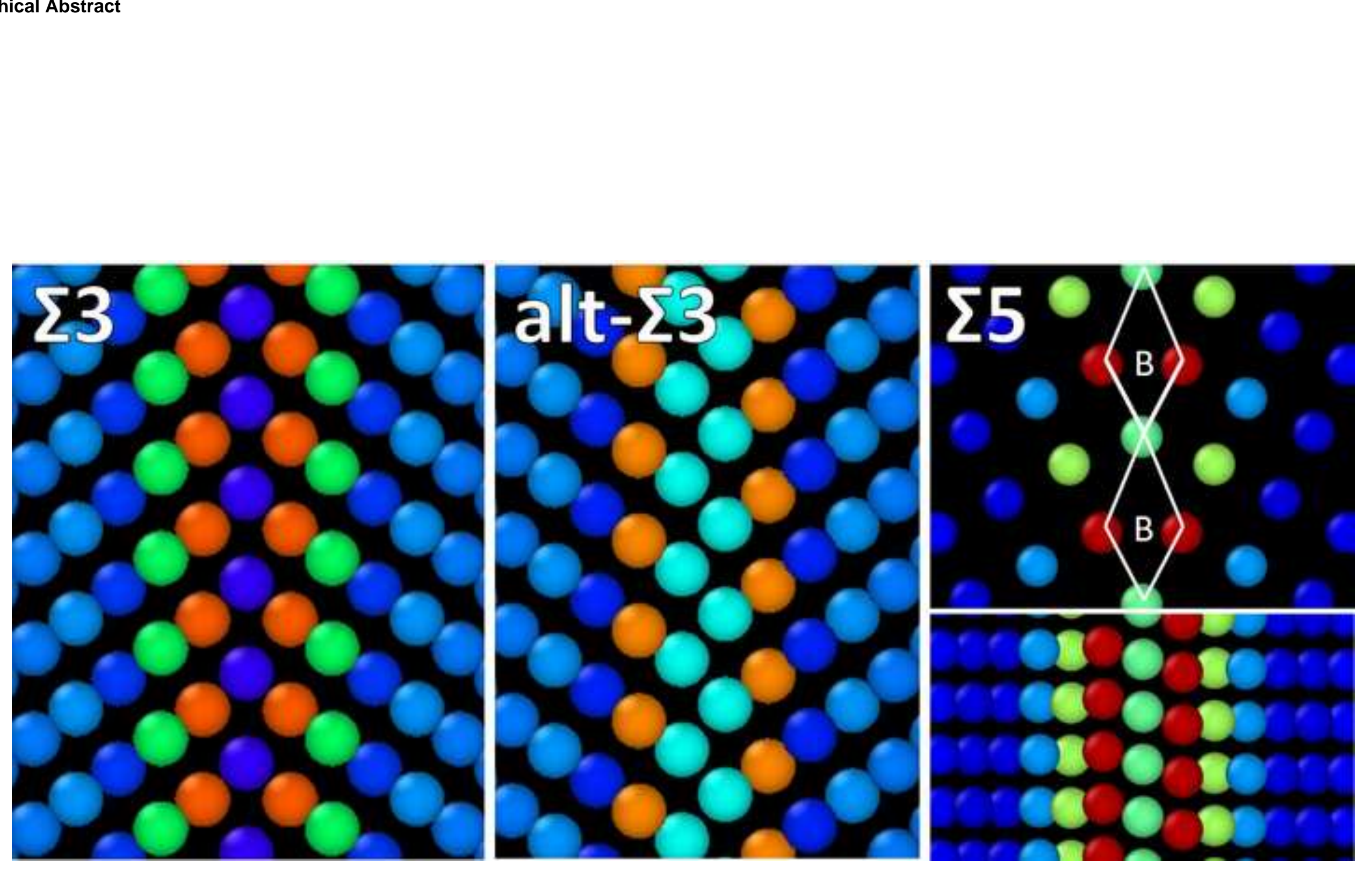

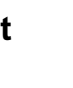
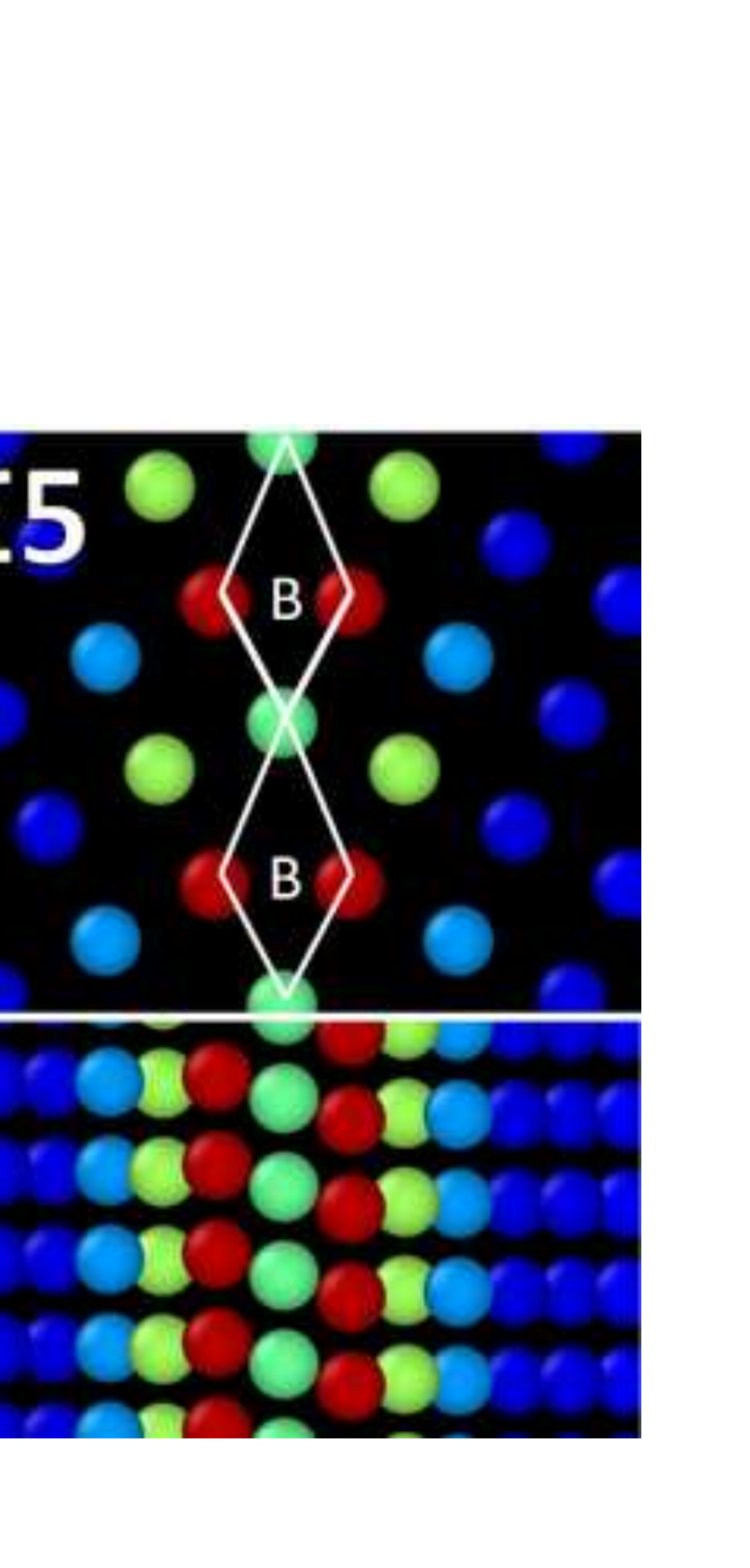


\title{
Symmetric Tilt Boundaries in Body-Centered Cubic Tantalum
}

\author{
Eric N. Hahn ${ }^{1,2, *}$, Saryu J. Fensin ${ }^{2}$, Timothy C. Germann ${ }^{2}$, Marc A. Meyers ${ }^{1}$ \\ ${ }^{1}$ University of California San Diego, La Jolla, CA 92093, USA \\ ${ }^{2}$ Los Alamos National Laboratory, Los Alamos, NM 94550, USA \\ *Corresponding author: enhahn@eng.ucsd.edu
}

\begin{abstract}
Grain boundaries can play a significant role in the mechanical response of materials. Atomistic simulations are used to investigate 79 coincidence site lattice grain boundary structures and energies in tantalum, a model body-centered cubic transition metal. Quasi-symmetric $\Sigma 3, \Sigma 5, \Sigma 7, \Sigma 13$, and $\Sigma 27$ boundaries are observed, of which $\Sigma 3$ and $\Sigma 7$ also exist as traditional mirror-symmetry conserving boundary structures. These results are supported by previous observations of similar phenomena in other bcc transition metal $\Sigma 5$ boundaries. Metastable low energy $\Sigma 3$ boundary structures in tantalum could influence the formation and stability of deformation twins and abnormal growth grain favoring $\Sigma 3$ boundaries.
\end{abstract}

KEYWORDS: coincidence lattice; grain boundary structure; grain boundary energy; molecular dynamics (MD); transition metals 
Grain boundaries play a critical role in the determination of mechanical, chemical, and thermal properties of polycrystalline materials. Specifically, the internal GB structure and energy can strongly determine grain boundary stability and influence the deformation response by affecting dislocation nucleation, dislocation motion, grain boundary sliding, diffusion, and radiation damage processes [1-7]. The distribution and character of grain boundaries is critical in controlling the strength of metals [8], especially tantalum [9-11]. The nature of grain boundaries is inherently complex because their energy is dependent on their character, which depends on five degrees of freedom. To these, one could add translation, which also changes the nature of the boundaries and has been shown to be particularly important for bcc metals [12]. A number of analytical approaches have been developed to treat GB structures: coincidence site lattice (CSL), displacement shift complete, and other topological treatments e.g., [13]. To investigate the dependence of deformation mechanisms on GB structural details, we first must understand the structural and energetic landscape of GBs as in recent surveys of face-centered cubic (fcc) [14] and some body-centered cubic (bcc) [15] materials.

Supporting these surveys, there have been numerous reports using both simulations and experiments to understand the GB structure and energies of fcc materials [14,16,17]. These studies have included both symmetric and asymmetric tilt boundaries on (111), (100), (110) and (113) planes [14,18]. In contrast, only a handful of studies exist on bcc transition metals including the work of Wolf on Fe/Mo [12,19], Yeşilleten and Arias on Mo [20], Ratanaphan et al. on Fe/Mo [15], and Shibuta et al. on Fe [21]. There are few experimental and/or simulation reference points for atomistic GB structures in tantalum; the principal reference is the structure of the $\Sigma 5(310) /[001]$ CSL tilt boundary investigated both experimentally and theoretically by Campbell et al. [22,23]. Hence, there is clearly a deficiency in available data for GBs in Ta within the current literature.

In the present work, we investigate GB structures in bcc tantalum and their associated energies using molecular dynamics (MD). Seventy-nine grain boundaries of varying tilt axis and misorientation (as described by the CSL model) are evaluated. The accompanying 'Data in Brief' details complete tilt axis $\langle a b c\rangle$, grain boundary normal $(h k l)$, CSL $\Sigma$ index, misorientation, and conversion of these values into appropriate simulation basis for four tilt axes: $\langle 001\rangle,\langle 011\rangle,\langle 111\rangle$, and $\langle 112\rangle$. We use the MD code LAMMPS [24] to generate and relax bicrystal structures based on the scheme of Tschopp et al. $[18,25,26]$. Briefly, within a fully periodic system, one crystal is sequentially shifted with respect to another along its $\gamma$-surface; atoms that exceed an overlap criteria are selectively removed; and the boundary is relaxed in the GB normal direction. The grain boundary energy (GBE) is calculated by evaluating the excess energy of the system per grain boundary unit area, taking into account that each simulation contains two parallel grain boundaries. The embedded atom model (EAM) potential developed 
by Ravelo et al. [27] is principally employed, but the importance of the $\Sigma 3$ coherent twin boundary to deformation behavior warranted a "quantum accurate" investigation using a spectral neighbor analysis potential (SNAP) developed by Thompson et al. [28]. Depending on the boundary orientations, between hundreds and thousands of possible atomic arrangements containing up to fifty thousand atoms were sampled to produce each minimum energy configuration, thus neither density functional theory (DFT) nor extended use of SNAP is practical for the present study.

Figure 1 shows the calculated GBEs as a function of misorientation angle alongside previous results for other bcc elements. GB structures presented in subsequent figures are indicated by vertical dashed lines. Based on calculations of surface energies for bcc transition metals, the energy of tantalum interfaces are bracketed between iron and molybdenum [29]. We note that it is to be expected that GBEs calculated in this work will be slightly lower than those calculated by tight binding or ab-initio methods $[17,30]$.

Minimas in energy are observed at specific misorientations for each of the tilt axes similar to fcc materials. However, in our case, the most prominent minimum in energy is associated with the $\Sigma 3<110>$ boundary shown in bold in Fig 1b. The $\Sigma 3$ boundary is a coherent twin boundary in bec metals and can play a critical role in plasticity by determining the propensity to nucleate deformation twins. Pressure and shear can alter barriers to deformation, such as stacking fault energies and the Peierls-Nabarro stress; relative energy barriers effectively determine whether full or partial dislocations are produced to relieve strain and ultimately influence the kinetics of deformation.

Two GB structures for the $\Sigma 3$ twin boundary are obtained using EAM: a structure with a welldefined mirror plane, $\Sigma 3$ in Fig. 2 a, and an alternate structure with a broken mirror plane, alternate- $\Sigma 3$ (alt- $\Sigma 3$ ) in Fig. 2b. The alt- $\Sigma 3$ boundary is quasi-symmetric, i.e. grain normals are equal and opposite in sign, but crystallographic mirror symmetry across the GB plane is broken [32]. The alt- $\Sigma 3$ structure can be obtained by shearing the coherent $\Sigma 3$ boundary in the boundary plane. As a result, the boundary takes a zig-zag configuration (light blue atoms) which decreases its energy. In fact, the alt- $\Sigma 3$ has the lowest observed energy of $278 \mathrm{~mJ} / \mathrm{m}^{2}$ in comparison to $293 \mathrm{~mJ} / \mathrm{m}^{2}$ for the $\Sigma 3$ boundary. In order to verify the unique low energy structure of the alt- $\Sigma 3$ structure we also employed the SNAP. Fig $2 c$ shows the SNAP predicted alt- $\Sigma 3$ which also has a structure and energy similar to the one predicted by EAM, indicating that the alt- $\Sigma 3$ structure is not a potential-dependent artificial GB structure.

Higher energy $\Sigma 3$ boundaries $\left(1298 \mathrm{~mJ} / \mathrm{m}^{2}\right)$ are also identified when the GB plane is changed to (111) from $(11 \overline{2})$ (tilt: $<011>$ ). Ab-initio calculations of the low energy $\Sigma 3$ boundary in tungsten [33] and iron [34] only evidence fully symmetric structures, but such symmetry was reinforced by small system size in combination with a priori atom displacements and minimizations. Broken and/or conserved 

potentials owing to the strong influence of non-spherically symmetric d-orbitals.

Similarly, the $\Sigma 5$ boundary (Fig. 3) structure breaks mirror symmetry; this contrasts early work of Ochs et al. [35], which shows, using simulations, that the Ta $\Sigma 5$ GB has full mirror symmetry. The $\Sigma 5$ is composed of " $B$ " structural units analogous to those identified in Fe [6]. The presently calculated GBE of $1318 \mathrm{~mJ} / \mathrm{m}^{2}$ is markedly less than the previously predicted $1544 \mathrm{~mJ} / \mathrm{m}^{2}$ [35]. More recent calculations using model generalized pseudopotential theory (MGPT) do predict a break in mirror symmetry for this boundary [22]. Ab-initio work by Ochs et al. [35] does, however, identify other bcc transition metal elements (Mo, $\mathrm{W}$, and $\mathrm{Nb}$ ) that break mirror symmetry. For $\mathrm{Nb}$ and $\mathrm{Mo}$, there exist experimental highresolution transmission electron microscopy (HRTEM) evidence for both structures with conserved and broken symmetry [36,37]. The relative shift across the boundary identified here for Ta is $0.81 \AA$ (as measured by the vertical displacement between opposing red atoms in Fig. 3b) as compared to $\sim 0.78 \AA$ for Mo. Relative to the respective lattice parameter (3.304 $\AA$ for Ta and $3.147 \AA$ for Mo) the displacements are within $\sim 1 \%$ of one another. If instead the shift is measured as the distance between atomic planes projected across the boundary, the shift is $0.55 \AA$, showing superb agreement to the experimentally observed shift of $\sim 0.55 \AA$ in Ta [22] (the MGPT value is not explicitly stated [22]).

Other boundaries corresponding to various energy minimas in Fig. 1 or comparable structures within the literature were also explored. Figure 4 shows selected boundaries for each of the tilt axes. Here, the grain boundary units of $\Sigma 11$ and $\Sigma 13$ are expressly identified for comparison with other bcc metals. The structure of the $\Sigma 13\langle 001\rangle$ boundary is analogous to the HRTEM observations of boundaries in Mo by Morita and Nakashima [31] and the $\Sigma 11\langle 011\rangle$ structure agrees well with the density functional theory (DFT) calculations of $\Sigma 11$ boundary in iron [34]. A large majority of the $\langle 112\rangle$ tilt axis boundaries exhibit large (periodic) boundary unit cells such as that shown for the $\Sigma 11\langle 112\rangle$. There exist multiple other predicted boundary structures that break mirror symmetry such as the alt- $\Sigma 27$, alt- $\Sigma 7$ and alt$\Sigma 13\langle 111\rangle$. Notably, the $\Sigma 7$ boundary also exhibits both a symmetric and quasi-symmetric structure of nearly equivalent energies further suggesting that the phenomenon of symmetry breaking is prevalent for many boundaries in bcc transition metals.

The classic processing-structure-properties-performance materials relationship underscores the need to identify grain boundary structures and their energies. Here we show a large number of grain boundaries energies for tantalum, as a function of tilt axis and misorientation, in order to inform future studies such as those investigating abnormal grain growth [10], heterogeneous deformation of poly and nanocrystals [9,39], and deformation twinning [11]. Specifically, deformation twinning involves the nucleation of $\Sigma 3$ coherent twin boundaries through the movement of twinning dislocations. It is shown here that there is a decrease in energy associated with the formation of quasi-symmetric boundaries. In 

asymmetric, and quasi-symmetric components. The metastability of the quasi-symmetric alt- $\Sigma 3$ boundaries in bcc tantalum, identified using both EAM and SNAP interatomic potentials, may play a governing role in determining the active mechanism for plastic deformation via the twinning vs. slip transition. Previous experimental evidence has shown quasi-symmetric $\Sigma 5$ GBs boundaries in bcc $\mathrm{Nb}$, Mo, and Ta; in the present work we identify symmetry breaking $\Sigma 3, \Sigma 5$, and $\Sigma 7$ GBs (among many others) in Ta. These quasi-symmetric structures appear to be unique to bcc transition metals and are not explicitly reported for fcc GB structures.

\section{ACKNOWLEDGEMENT}

ENH and MAM were supported by UC Research Laboratories Grant (09-LR-06-118456-MEYM), and by the Department of Energy NNSA/SSAP (DE-NA0002080). ENH also received support from DOE ASCR through the Exascale Co-design Center for Materials in Extreme Environments. Los Alamos National Laboratory is operated by LANS, LLC, for the NNSA and the U.S Department of Energy under contract DE-AC52-06NA25396.

\section{REFERENCES}

[1] T. Frolov, D.L. Olmsted, M. Asta, Y. Mishin, Nat. Commun. 4 (2013) 1899.

[2] Y. Mishin, M. Asta, J. Li, Acta Mater. 58 (2010) 1117-1151.

[3] S.J. Fensin, E.K. Cerreta, G.T. Gray III, S.M. Valone, Sci. Rep. 4 (2014).

[4] S.J. Fensin, S.M. Valone, E.K. Cerreta, J.P. Escobedo-Diaz, G.T. Gray III, K. Kang, J. Wang, Model. Simul. Mater. Sci. Eng. 21 (2013) 015011.

[5] G.S. Rohrer, J. Mater. Sci. 46 (2011) 5881-5895.

[6] M.A. Tschopp, K.N. Solanki, F. Gao, X. Sun, M.A. Khaleel, M.F. Horstemeyer, Phys. Rev. B 85 (2012) 064108.

[7] G.J. Tucker, M.A. Tschopp, D.L. McDowell, Acta Mater. 58 (2010) 6464-6473.

[8] E.N. Hahn, M.A. Meyers, Mater. Sci. Eng. A (2015).

[9] T.R. Bieler, S.C. Sutton, B.E. Dunlap, Z.A. Keith, P. Eisenlohr, M.A. Crimp, B.L. Boyce, JOM 66 (2013) 121-128.

[10] N.A. Pedrazas, T.E. Buchheit, E.A. Holm, E.M. Taleff, Mater. Sci. Eng. A 610 (2014) 76-84.

[11] V. Livescu, J.F. Bingert, T.A. Mason, Mater. Sci. Eng. A 556 (2012) 155-163.

[12] D. Wolf, Philos. Mag. A 62 (1990) 447-464.

[13] A.P. Sutton, R.W. Ballufi, Philos. Mag. Lett. 61 (1990) 91-94.

[14] D.L. Olmsted, E.A. Holm, S.M. Foiles, Acta Mater. 57 (2009) 3704-3713.

[15] S. Ratanaphan, D.L. Olmsted, V.V. Bulatov, E.A. Holm, A.D. Rollett, G.S. Rohrer, Acta Mater. 88 (2015) 346-354.

[16] M.J. Mills, M.S. Daw, G.J. Thomas, F. Cosandey, Ultramicroscopy 40 (1992) 247-257.

[17] A.F. Wright, S.R. Atlas, Phys. Rev. B 50 (1994) 15248-15260.

[18] M.A. Tschopp, D.L. Mcdowell, Philos. Mag. 87 (2007) 3871-3892.

[19] D. Wolf, Philos. Mag. Part B 59 (1989) 667-680.

[20] D. Yeşilleten, T.A. Arias, Phys. Rev. B 64 (2001) 174101.

[21] Y. Shibuta, S. Takamoto, T. Suzuki, ISIJ Int. 48 (2008) 1582-1591.

[22] G.H. Campbell, J. Belak, J.A. Moriarty, Scr. Mater. 43 (2000) 659-664.

[23] G.H. Campbell, W.E. King, J. Belak, J.A. Moriarty, S.M. Foiles, in:, Symp. Q - Adv. Mater. Probl. Solving Electron Microsc., 1999. 
[24] S. Plimpton, J. Comput. Phys. 117 (1995) 1-19.

[25] M.A. Tschopp, G.J. Tucker, D.L. McDowell, Comput. Mater. Sci. 44 (2008) 351-362.

[26] M.A. Tschopp, G.J. Tucker, D.L. McDowell, Acta Mater. 55 (2007) 3959-3969.

[27] R. Ravelo, T.C. Germann, O. Guerrero, Q. An, B.L. Holian, Phys. Rev. B 88 (2013) 134101.

[28] A.P. Thompson, L.P. Swiler, C.R. Trott, S.M. Foiles, G.J. Tucker, J. Comput. Phys. 285 (2015) 316330.

[29] J.-M. Zhang, D.-D. Wang, K.-W. Xu, Appl. Surf. Sci. 252 (2006) 8217-8222.

[30] T. Ochs, C. Elsässer, M. Mrovec, V. Vitek, J. Belak, J.A. Moriarty, Philos. Mag. A 80 (2000) 24052423.

[31] K. Morita, H. Nakashima, Mater. Sci. Eng. A 234-236 (1997) 1053-1056.

[32] A. Morawiec, Z. Für Krist. Cryst. Mater. 227 (2012) 199-206.

[33] L. Zhi-Wu, K. Xiang-Shan, Liu-Wei, L. Chang-Song, F. Qian-Feng, Chin. Phys. B 23 (2014) 106107.

[34] S.K. Bhattacharya, S. Tanaka, Y. Shiihara, M. Kohyama, J. Phys. Condens. Matter 25 (2013) 135004.

[35] T. Ochs, O. Beck, C. Elsässer, B. Meyer, Philos. Mag. A 80 (2000) 351-372.

[36] G.H. Campbell, J. Belak, J.A. Moriarty, Acta Mater. 47 (1999) 3977-3985.

[37] G.H. Campbell, S.M. Foiles, P. Gumbsch, M. Rühle, W.E. King, Phys. Rev. Lett. 70 (1993) 449-452.

[38] A.P. Sutton, V. Vitek, Philos. Trans. R. Soc. Lond. Math. Phys. Eng. Sci. 309 (1983) 55-68.

[39] C.H. Lu, B.A. Remington, B.R. Maddox, B. Kad, H.S. Park, M. Kawasaki, T.G. Langdon, M.A. Meyers, Acta Mater. 61 (2013) 7767-7780.

Figure 1. Grain boundary energy as a function of misorientation for four tilt axis. GBEs for Ta are shown as black asterisks (GBE values can be found in the supplemental material). Also provided is relevant data for bcc Fe (blue) and bcc Mo (red) from empirical potentials (Wolf [12,19], Morita and Nakashima [31], and Tschopp et al. [6]) illustrating similar trends.

Figure 2. Configurations of $\Sigma 3\langle 011\rangle$ boundary colored by eV/atom shown for two projections. In each projection the GB normal is horizontal. (Left) EAM symmetric twin boundary. (Middle) EAM quasisymmetric boundary with broken mirror symmetry in both (110) and (111) projections. (Right) SNAP quasi-symmetric boundary.

Figure 3. $\Sigma 5$ boundary visualization with color according to atomic potential energy in eV/atom. (Top) Boundary structural unit $B$ identified in the typical fashion [38]. (Bottom) Projection illustrating broken mirror symmetry shift.

Figure 4. Assorted low energy grain boundary structures for each of the four tilt axis with equivalent coloring scheme as Fig. 3. From left to right the boundaries are: $\Sigma 13(051)\langle 001\rangle, \Sigma 11(332)\langle 011\rangle$, $\Sigma 27(552)\langle 011\rangle, \Sigma 7(231)\langle 111\rangle$, alt- $\Sigma 7(231)\langle 111\rangle, \Sigma 13(341)\langle 111\rangle, \Sigma 11(131)\langle 112\rangle$. 

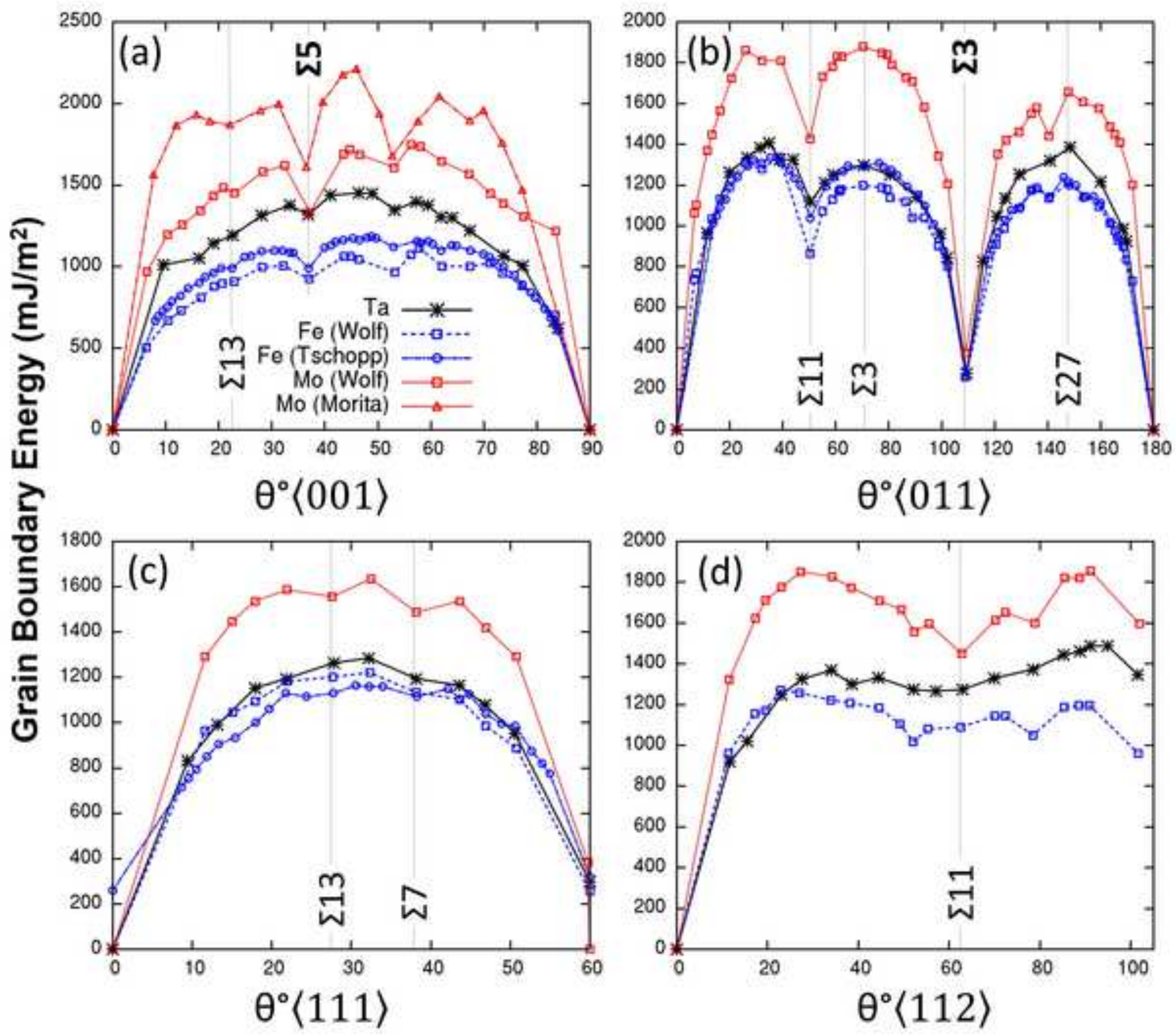


\section{(a) $\Sigma 3$ (EAM)}
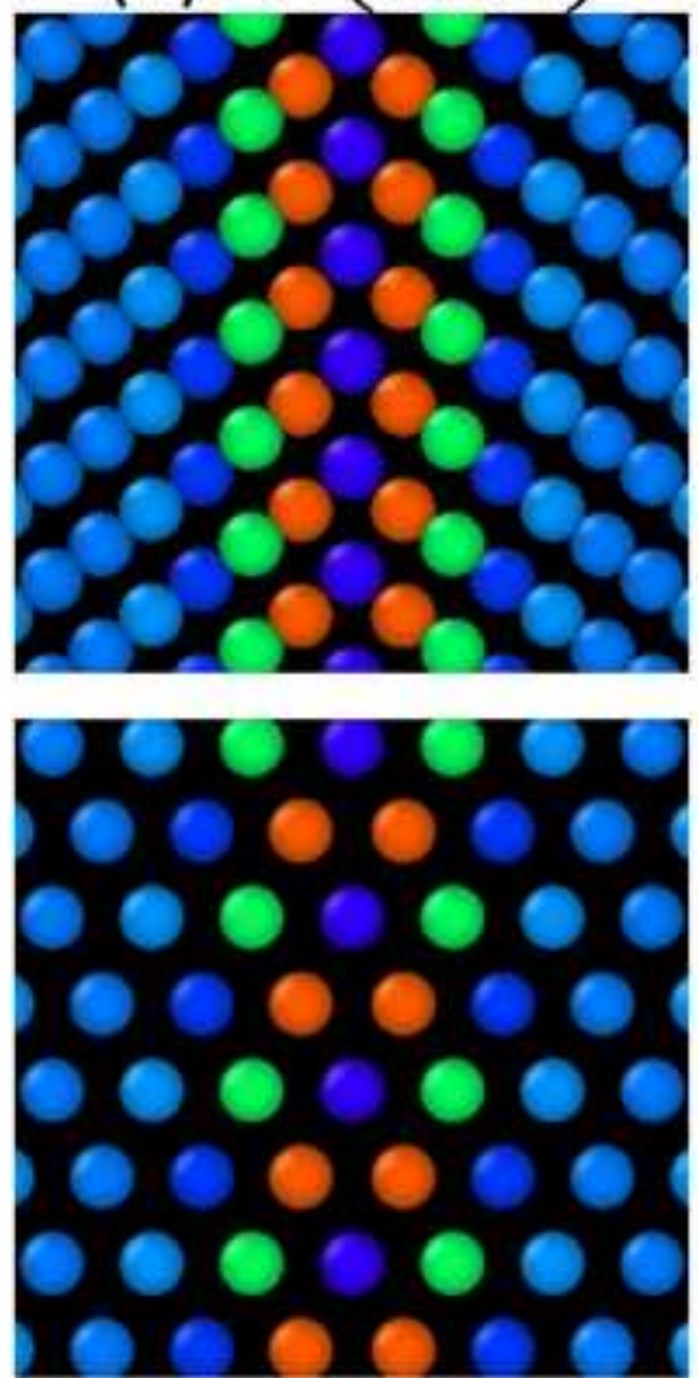

(b) alt- 53 (EAM)
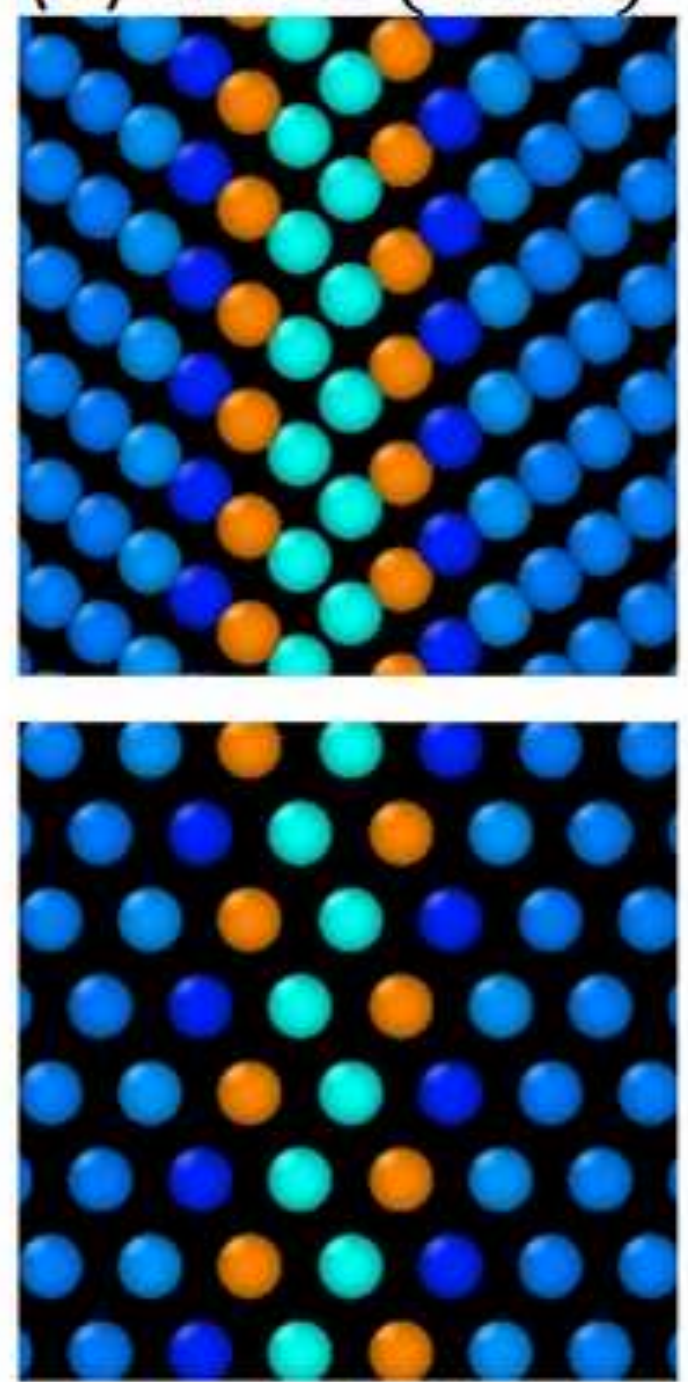

(c) alt- $\Sigma 3$ (SNAP)

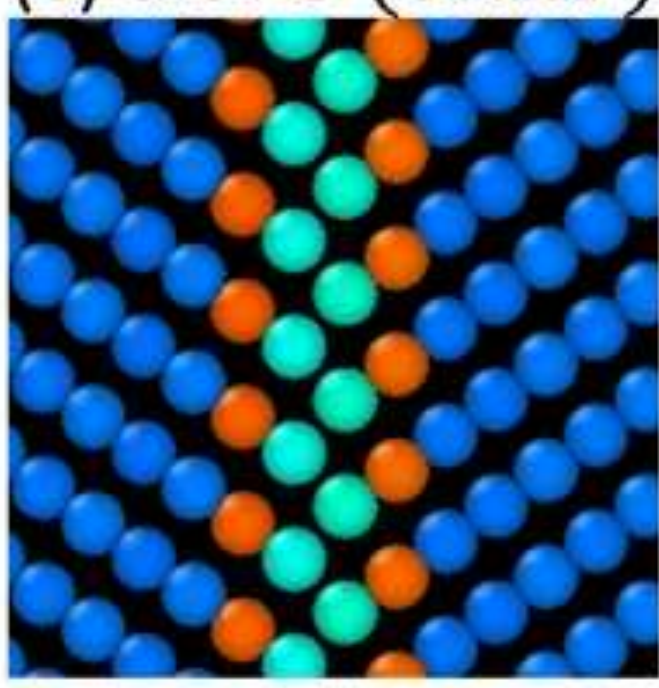

$-8.125$

กิ (111)

$\exists$
ลิ่ $(\overline{1} 10)$

$\exists$

$-7.975$
$\mathrm{eV}$

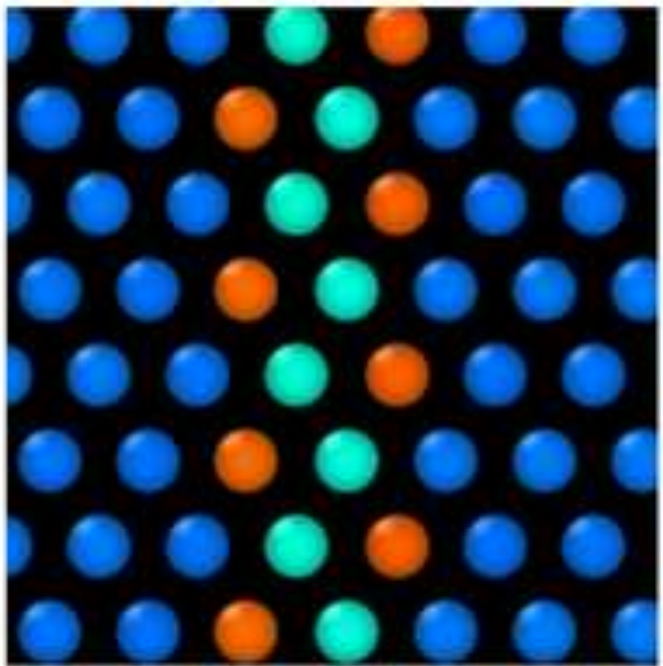

1000000

000000

bogoen 


\section{$\Sigma 5(031)\langle 001\rangle$}
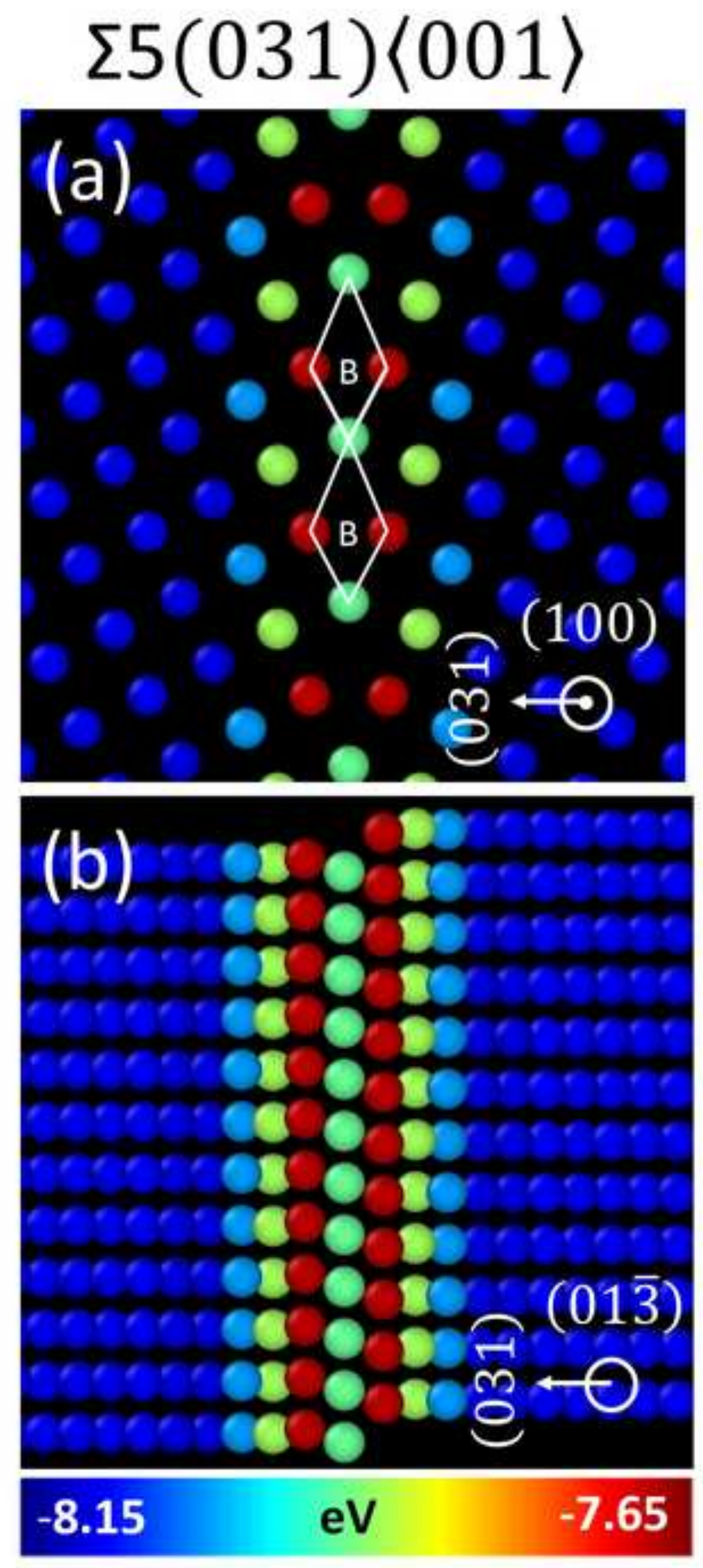

Figure(s) 

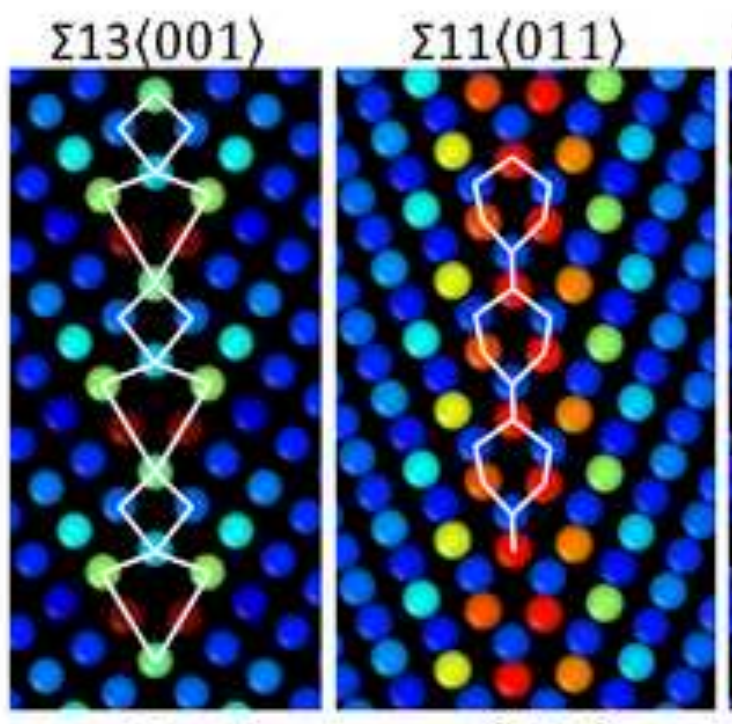

$$
\text { ㄷํㅇ }
$$

ลิ (011)

m -0

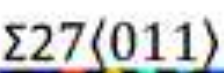

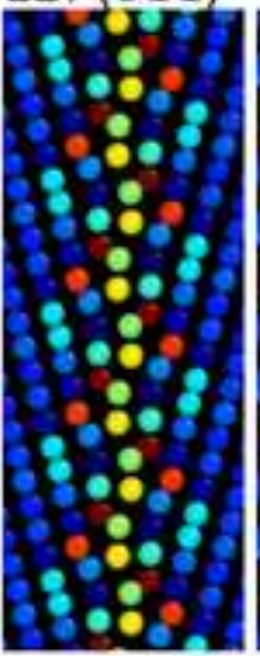

ลิ (011)

은

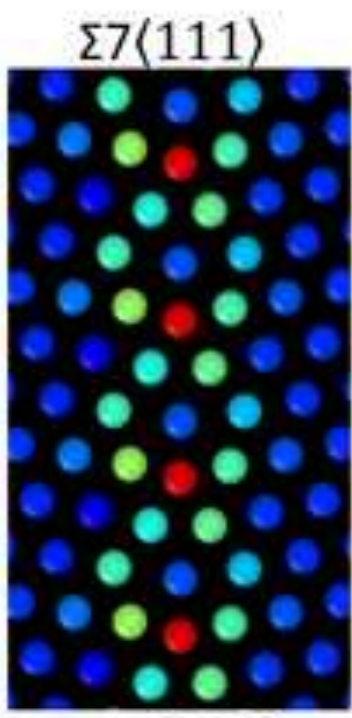

곡

iv - alt- $\sum 7\langle 111\rangle$

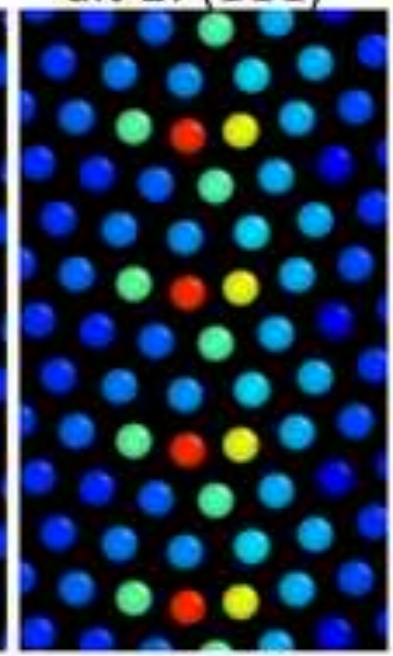

ㄱ (111)

กำ
513〈111)

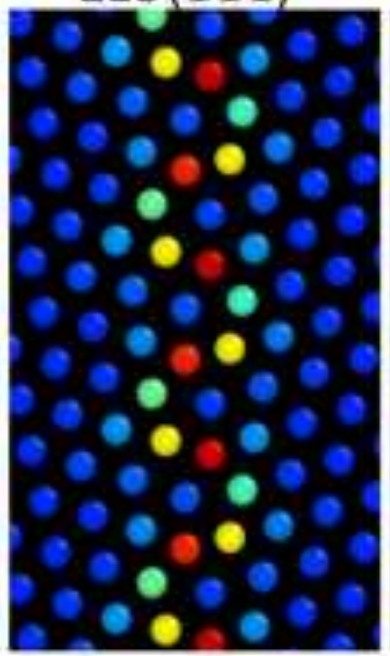

I (111)

ले
$\Sigma 11\langle 112\rangle$

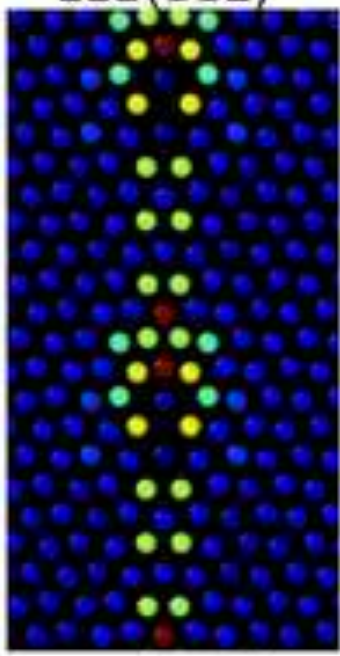

(112)

$\stackrel{m}{-} \odot$ 